\title{
Comparative Review of Drugs Used in Diabetes Mellitus-New and Old
}

\section{Furqan Ul Haq1, Abuzar Siraj ${ }^{2}$, Muhammad Atif Ameer ${ }^{3}$, Tanveer Hamid ${ }^{4}$, Mansoor Rahman', Salman Khan', Saad Khan6, Sarwat Masud7}

${ }^{1}$ Hayatabad Medical Complex Peshawar, Peshawar, Pakistan

${ }^{2}$ Shaukat Khannum Memorial Hospital and Research Centre, Peshawar, Pakistan

${ }^{3}$ Medical Research Centre, University of Nebraska, Nebraska, USA

${ }^{4}$ Saidu Group of Teaching Hospitals, Swat, Pakistan

${ }^{5}$ Lady Reading Hospital, Peshawar, Pakistan

${ }^{6}$ Khyber Teaching Hospital, Peshawar, KP, Pakistan

${ }^{7}$ Agha Khan University, Karachi, Pakistan

Email: furqanulhaq459@gmail.com

How to cite this paper: Ul Haq, F., Siraj, A., Ameer, M.A., Hamid, T., Rahman, M., Khan, S., Khan, S. and Masud, S. (2021) Comparative Review of Drugs Used in Diabetes Mellitus-New and Old. Journal of Diabetes Mellitus, 11, 115-131. https://doi.org/10.4236/jdm.2021.114009

Received: April 25, 2021

Accepted: September 10, 2021

Published: September 13, 2021

Copyright $\odot 2021$ by author(s) and Scientific Research Publishing Inc. This work is licensed under the Creative Commons Attribution International License (CC BY 4.0).

http://creativecommons.org/licenses/by/4.0/

\begin{abstract}
Background: Diabetes mellitus (DM) is a syndrome of chronically elevated glucose level in the blood either due to insulin resistance, insulin deficiency or both. In addition, it may occur due to defective metabolism of carbohydrates, fats and proteins. There are 3 main types of DM: Type $2 \mathrm{DM}$ is more prevalent in adults and is typically due to relative insulin deficiency, deficiency of insulin in children leads to DM type 1; and lastly, gestational diabetes occurs during pregnancy resulting from an imbalance of placental hormones. Introduction: Insulin, Biguanides and Sulfonylureas are some of the drug classes used to treat DM. However, their use is complicated by numerous side effects, such as; hypoglycemia \& weight gain from insulin and sulfonylureas; lactic acidosis, vitamin B12 deficiency and gastrointestinal upset with metformin. Route of administration and cost are also important factors to consider when prescribing. It is for this reason the quest for newer, safer and easier to administer drugs is ongoing. Methodology: Used all the articles available on anti Diabetic drugs on web especially in British Medical Journal, Elsevier, Pubmed, Google scholar and Wikipedia etc. Got a final review article to compare the older and newer anti Diabetic drugs. Results and Conclusion: Insulin is good for controlling acute hyperglycemic states in DM but it causes acute hypoglycemia and lipodystrophy. Metformin is good hypoglycemic and easily available but causes hypoglycemia, metallic taste, Lactic acidosis and B12 deficiency. Sulfonylureas are good hypoglycemic but causes severe hypoglycemia acutely and weight gain so contraindicated for obese or
\end{abstract}


hypertensive patients. While newer antidiabetics such as GLP 1 agonists increases insulin secretions has very low risk of hypoglycemia, causes weight loss as compared to insulin and decreases risk of cardiovascular side effects but still can't be used in renally impaired patients, causes pancreatitis and can not be given in gastroparesis patients, similarly a newer drug of this class known as LY2189265 has long halflife of 90 hours, better efficacy, but causes pancreatitis and increase diastolic BP in high doses, pancreatitis is not associated with lixisenatide (GLP 1 agonist), while DPP4 inhibitors which increases GLP 1 in body has less risk of hypoglycemia, GI side effects, are weight neutral can be used in CKD but causes headaches and Nasopharyngitis. Bromocriptine or pegvisomant are used in patients of growth hormones adenoma induced DM as a medical therapy but are associated with psychosis and hallucinations. Meglitinides increases insulin secretion and has minuscule risk of hypoglycemia but can not be used in CKD patients. Otelixizumab and Teplizumab decrease $\mathrm{T}$ cell functions and save beta cells from immune reactions used in DM 1 but cause immune suppression and is an orphan drug. Recombinant GAD used in vaccines decreased antibody mediated beta cell damage but is still under studies.

\section{Keywords}

Anti-Diabetic Drugs, Metformin, Sitagliptin, Canagliflozin, Exenatide, Pioglitazone, Insulin Use and Its Efficacy, Glipizide

\section{Introduction \& Background}

Diabetes Mellitus (DM) is a metabolic syndrome occurring due to relative or absolute insulin deficiency which results in a chronically elevated blood glucose level and has polyuria, polyphagia and polydipsia. There are many types of DM discussed below, all of them present at specific age and specific conditions.

1) Type $1 \mathrm{DM}$ is due to beta cells dysfunction from attack of auto antibodies against beta cells of pancreas, and it is destroyed by antibodies, B cells and $\mathrm{T}$ cells. This type of DM presents mostly in early age of life especially in females children.

2) Type 2 DM occurs due to insulin ineffectiveness or resistance and because of that Insulin doesn't work on its own receptors to lower the glucose level.

3) Genes responsible for Beta cell functions: Maturity onset diabetes mellitus of young (MODY) and other insulin gene mutations.

4) Genetic defects in insulin action such as receptors mutations will also lead to DM.

5) Exocrine pancreatic defects such as in chronic pancreatitis, cystic fibrosis, hemochromatosis such conditions always presents with hyperglycemia in chronic stages.

6) Endocrine disorders such as in Cushing syndrome and growth hormone 
excess as it increases glucose levels and causes DM.

7) Viruses such as in CMV, Coxsakievirus B or congenital Rubella.

8) Drugs such as glucocorticoids, beta adrenergic agonists usage for chronic conditions causes DM.

9) Gestational diabetes mellitus occurs in pregnancy especially 2nd and 3rd trimester due to human placental Lactogen leads to insulin resistance and hyperglycemia [1].

It goes without saying that due to the vast nature of the disease, the type of drug used varies from patient to patient. This is what we aim to highlight in this review.

\section{Epidemiology}

The Global prevalence of diabetes mellitus in 2019 was 9.3\% (463 million people) will be $10.2 \%$ (578 million) in 2030 and $10.9 \%$ (700 million) in 2045 , if prevalence increases with the same rate [2]. In USA, $10.5 \%$ (34.2 million) people have diabetes, of which $95 \%$ is DM type 2 in adults, as it makes $13 \%$ of diabetes mellitus type in people of age 18 or older [3].

Treatment Strategies of Diabetes Mellitus:

In addition to advising lifestyle modifications such as exercise, decreasing consumption of carbohydrate, fats, increase in protein rich diet and monitoring for micro- and macro-vascular complications, patients with specific types of DM are treated with the following:

- Type 1 DM: It is treated by insulin replacement.

- Type 2 DM: It is treated Metformin and sulfonylureas if severe than add insulin.

- Gestational Diabetes: Its treated with Insulin and/or metformin in select cases [4].

Drug classes:

1) Insulin and its different types.

2) Biguanides (metformin).

3) Sulphonylureas.

4) Glucagon like peptide 1 analogues: Exenatide, Liraglutide.

5) Dipeptidyl peptidase 4 inhibitors: Linagliptin, Sitagliptin, Saxagliptin.

6) Peroxisomal proliferator activating receptor gamma inhibitors: pioglitazone, rosiglitazone.

7) Alpha Glucosidase inhibitors: Acarbose and Miglitol.

8) Meglitinides: Repaglinide, Nateglinide.

9) Sodium Glucose co-transporter 2 inhibitors: Canagliflozin, Empagliflozin, Dapagliflozin.

10) Amylin analogues: Pramlintide.

11) Monoclonal antibodies and secondary drugs: Bromocriptine, Otelixizumab, Recombinant human Glutamic acid Decarboxylase 65 (rhGAD65), Succinobucol, Teplizumab, LY2189265, AVE0010/ZP10 [5]. 


\section{Anti Diabetic Drugs Classes}

\subsection{Insulin and Its Types}

There are multiple types of insulin available. The four main types are:

1) Rapid-acting insulin

2) Regular-acting or short-acting insulin

3) Intermediate-acting insulin

4) Long-acting insulin

Routes of administration are Bucal, Transdermal, pulmonary as in inhalers. In inhaler form, the drug reaches the polmunary capillaries and polmunary veins and absorbs there and joins the circulations.

\subsection{Other Novel Methods of Insulin Usage}

Red blood cells, found in blood, are the contributing majority of cells in the body and can carry many drugs very easily to every part of the body, directly or by diffusion, and in those drugs one is insulin. Since RBCs has long half life approximately upto 120 days, so it's proving long halflife for drugs, increases bioavailability almost of $100 \%$, so considered as an excellent carriers. There are high molecular weight proteins as macromolecules, in 3D structured used for delivery of drugs attached to its chains. Dendrimers are also used for successful delivery of insulin [5].

Rapid acting insulin products are:

Lispro, Aspart, Glulisine

Regular acting Insulin products are:

Humulin, Iletin, Novolin, Relion, Velosulin

Intermediate acting Insulin products are

Neutral protamine Hagedron or NPH or Isophane.

Long acting Insulin products are:

Detemir, Degludec, Glargine.

\subsection{Mechanism of Action of Insulin in Lowering Glucose Level in Blood}

Insulin Binds insulin receptor which is having tyrosine kinase activity.

1) in hepatocytes the insulin increases conversion of glucose to glycogen.

2) in skeletal Muscle or smooth: it increases glycogen as well as protein synthesis.

3) in Fatty tissues: increase Triglycerides synthesis and accumulation.

4) Increases potassium uptake by the cells as in general.

Adverse effects:

Insulin can cause hypoglycemia acutely, local lipodystrophy, hypersensitivity reactions (rarely) and weight gain.

\subsection{Biguanides}

Metformin, Buformin and Phenoformin, Proguanil, Chlorproguanil, Chlorhex- 
idine, Polyaminopropyl biguanide and Polyhexanide.

Metformin is taken orally well absorbed from gastrointestinal tract and it is excreted from body renally unchanged. Widely used in diabetes mellitus type 2,not used in type $1 \mathrm{DM}$ causes hypoglycemia because Lowers gluconeogenesis in liver, increase peripheral glucose intake by increasing insulin sensitivity and increases glycolysis, however in hyperinsulinemia during fasting Biguanides decreases Insulin level in blood because it increases insulin induced glucose uptake, so less blood glucose will cause feedback inhibition of insulin release.

Proguanil and chlorproguanil are biguanides used as antimalarials. Chlorhexidine and polyhexanide are disinfectants.

It causes Gastrointestinal upset such metallic taste, anorexia, nausea, vomiting, diarrhea and Dyspepsia in almost one third of patients, decreases vitamin B12 absorption, Lactic acidosis so shall not be used in renally impaired patients or chronic respiratory disease patients because they won't be able to compensate for acidosis and also causes weight loss. Buformin and Phenoformin are withdrawn from the market due to severe toxicity such as Lactic acidosis. It is contraindicated in patients of Alcoholism and hepatic insufficiency. Weight loss and insulin sensitivity increasing property is helpful in polycystic ovarian syndrome and obese patients. Lower price and easily available on different brand names such as Glucophage the rest of drugs are not used these days, they are withdrawn from market such as Buformin and Phenoformin.

\subsection{Sulfonylureas: (Insulin Secretagogues Just Like Meglitinides and D Phenylalanine Derivatives)}

1st generation:

Chlorpropamide (long acting), Tolbutamide (short acting), Acetohexamide, Carbutamide, Glycinamide, Metahexamide, Tolazamide.

2nd generation:

Glipizide, Glimepride, Glibenclamide, Gliburnuride, Glyclazide, Gliquidone, Glisopexide and Glyclopyramide.

It closes the potassium channel present on pancreatic beta cells membranes and so cell membrane depolarizes and $\mathrm{Ca}$ will go into cells and binds to insulin containing vesicles causes its degradation and causing increased release of insulin [6].

Post pancreatectomy or patient of Diabetes Mellitus type one having absolute deficiency of beta cells, Sulfonylureas will not be effective in those patients. Its 1 st generation causes Disulfiram-like reactions such as by Chlorpropamide and Tolbutamide. Generally Sulfonylureas are associated with hypoglycemia and weight gain especially in renally impaired patients or diabetic patients while fasting.

Its action is enhanced by drugs which decrease its renal clearance such as $\mathrm{Al}$ lopurinol and acetylsalicylic acid, cytochrome p450 inhibitors also enhance its action such as Sulphonamides, fibrates. Some drugs lower its hypoglycemic effects such as sympathomimetic like Salbutamol, Terbutaline, Dobutamine etc., 
Isoniazid, Glucocorticoids, oral contraceptive pills and thyroid hormones. Due to these wide range of drug interactions, it's not always a safer choice for Diabetic patients with comorbidities such as infections, Tuberculosis and Cardiovascular and pulmonary diseases.

According to Cochrane review in 2014 they studied with evidence that people treated with SU were less likely to develop cardiovascular side effects than with Metformin but SU are associated with higher risk of hypoglycemia (RR 5.6) than hypoglycemia with Metformin (RR 0.60). It's easily available in the market and at lower price.

\subsection{Glucagon-Like Peptide 1 (GLP1) Receptor Agonists}

GLP 1 is physiologically released in response to oral meals. It has receptors present on many tissues throughout the body. But their effect on the gastrointestinal tract is what makes them suitable for DM type 2. In Beta cells of the pancreas, GLP 1 causes increased glucose-mediated release of insulin. It also decreases glucagon release after meals. It also slows gastric emptying.

GLP 1 is degraded by the enzyme dipeptidyl peptidase 4 (DPP4), which are targets of the -gliptin family of diabetes drugs. Hence, the half life of GLP1 analogue ranges from 1 to 2 minutes. Synthetic analogues of GLP 1 (exenatide, liraglutide, albiglutide, taspoglutide, lixisenatide, dulaglutide) are relatively resistant to degradation by DPP4 [7].

Similar to insulin, GLP 1 analogues are injectable drugs. But in stark contrast, they result in weight loss rather than weight gain, which is a much undesirable side effect of insulin [8]. In addition, the risk of hypoglycemia is miniscule compared to insulin [9].

Due to the progressive course of type 2 diabetes mellitus, most patients require intensification of treatment regimens. That being said, the first line therapy still remains weight loss, lifestyle modification and metformin. GLP 1 agonists are not the first line for type $2 \mathrm{DM}$, and are avoided altogether in type $1 \mathrm{DM}$.

GLP agonists have been shown to considerably reduce HBA1c levels. However, arguably their most important effect is reduction in cardiovascular disease outcomes in patients with type 2 diabetes. It significantly decreased the rate of death from cardiovascular causes, nonfatal myocardial infarction and nonfatal stroke [10] [11].

Though their efficacy is quite apparent, GLP agonists do have certain side effects. The most commonly reported are nausea, vomiting and diarrhea. Acute pancreatitis has also been associated with GLP 1 agonist use. Since it is an injectable drug, injection site reactions have been reported. More importantly, GLP 1 agonists should not be used in patients with renal dysfunction particularly if creatinine clearance is below $30 \mathrm{~mL} / \mathrm{min}$ [12] Gastroparesis may be exacerbated by GLP 1 agonists as they cause further increase transit time through the stomach. Affordability may also be an issue, as these drugs are relatively expensive.

With the above in mind, GLP 1 agonists are suitable drugs for type 2 diabetics 
with cardiovascular disease, or if hypoglycemia is to be avoided, or when weight gain due to other diabetic drugs is complicating management.

\subsection{Sodium-Glucose Co-Transporter 2 (SGLT2) Inhibitors}

SGLT2 receptors are found on the proximal convoluted tubules of the kidney. They are responsible for reabsorbing $90 \%$ of the filtered glucose. The drugs that inhibit this transporter (dapagliflozin, empagliflozin, canagliflozin) reduce blood glucose levels by inducing osmotic diuresis. This is especially important because the diuretic effect is limited by the amount of filtered glucose, which in turn is dictated by the blood glucose level. Because of this, SGLT2 inhibitors do not cause hypoglycemia.

However, SGLT2 inhibitors are not first line agents for type 2 diabetes. They are only added if adequate glycemic control cannot be achieved with lifestyle modifications and metformin. In addition, empagliflozin and canagliflozin are particularly useful in decreasing morbidity and mortality in type 2 diabetics with established athersclerotic cardiovascular disease [13]. All SGLT2 inhibitors are useful in patients with heart failure as they reduce the risk of heart failure exacerbation requiring hospitalization and cardiovascular death. That being said, they are not the most effective at improving glycemic control, and so are frequently used in combination with other antidiabetic agents. SGLT2 inhibitors have shown to decrease the progression of diabetic nephropathy, so used in patients with albumin-creatinine-ratio greater (ACR) than 300 or estimated glomerular filtration rate (eGFR) less than $90 \mathrm{~mL} / \mathrm{min} / 1.73 \mathrm{~m}^{2}$. However, their benefit is limited in patients with very low eGFR (less than $30 \mathrm{~mL} / \mathrm{min} / 1.73 \mathrm{~m}^{2}$ )

SGLT2 inhibitors may also cause weight loss, which is a beneficial effect as most other antidiabetic drugs can weight gain, which complicates management of diabetes [14]. However, they don't come without side-effects. SGLT2 inhibitors have been implicated in causing diabetic ketoacidosis (DKA), particularly euglycemic DKA which results in delayed recognition and treatment [15]. This could be due to the volume depletion caused by these drugs, which also results in decreased blood pressure. This may result in orthostasis which results in falls. Compounded by the reduced bone mineral density, patients on SGLT2 inhibitors have increased rates of bone fractures. The glucose-rich urine is also responsible for causing genitourinary infections, especially vulvovaginal candidiasis in women. Moreover, canagliflozin is particularly implicated in increasing the rate of amputation that's why it has been black boxed warned by FDA. Especially in patients with neuropathy, foot deformity, peripheral arterial disease and history of prior foot ulceration.

Keeping all of the above in mind, patients on SGLT2 inhibitors should be monitored with regular HBA1c and fasting blood glucose level measurement, renal function with eGFR and blood pressure for volume status.

\subsection{Alpha Glucosidase Inhibitors}

An alpha-glucosidase inhibitor is a group of anti diabetic drugs that are used for 
the management of DM 2. Alpha-glucosidase inhibitors can be used alone or with combinations of other drugs' anti-diabetics regime. AGI is approved for type 2 diabetes Mellitus only by the FDA, but it is also effective in type $1 \mathrm{DM}$ and gestational DM [16]. The most prominent indication for using AGI is postprandial hyperglycemia. They modestly decrease glycosylated hemoglobin levels and also helps in reducing postprandial insulin concentrations [17]. AGI also reduces glucose variability throughout the day as compared to other oral hypo glycemics. As the postprandial directly correlates to the microvascular complication, so it plays a vital role. Moreover, Acarbose has proven efficacy in stabilizing carotid plaques in diabetic patients and has been shown to improve patients' life expectancy with diabetes mellitus type 2 by reducing cardiovascular complications [18].

The primary MOA of AGI is delaying and inhibiting the absorption of carbohydrates from small intestines. AGI competitively inhibit enzymes (sucrase, maltase, iso-maltase and glucoamylase) in the brush border of the enterocytes which are involved in converting non-absorbable oligo and oligosaccharides into simple absorbable monosaccharides. By delaying absorption of carbohydrates in the gut, AGI decrease the rise of blood glucose levels around $3 \mathrm{mmol} / \mathrm{L}$ post prandial.

Miglitol is absorbed from the gut and is excreted through the urine. Out of all Acarbose is the most widely studied and used drug. It specifically inhibits alpha-amylase, maltase, sucrase, dextranase, and glucoamylase. It is best studied for late dumping syndrome. Moreover, Acarbose has potential to prevent beta-cell hypertrophy, beta cell hyperplasia, and hyperinsulinemia hypoglycemia [19]. Acarbose and voglibose are absorbed poorly from the gut, have very low bioavailability and both of them are secreted in the stool. The effectiveness of the AGI varies with each patient and depends on the quantity of the complex carbohydrates consumed by the patient. Alpha glucosidase inhibitors are consumed orally and are the first line of drug in type 2 diabetes mellitus. AGI can be used as a first line drug in the patient who are newly diagnosed with DM type 2 which is initially treated with exercise and diet. AGI can be taken three times a day right before the consumption of a meal. It is recommended to always start from a lower dose to avoid hypoglycemia and also to titrate for the optimal dose periodically. Higher dose taken initially can lead to hypoglycemia which can be fatal if the patient is not in the hospital settings. The maximum dose of Acarbose is $100 \mathrm{mg}$ taken thrice a day and the lowest dose is $25 \mathrm{mg}$ thrice a day. The maximum dose of miglitol is also $100 \mathrm{mg}$ taken three times daily 6 hours apart and the lowest dose is $25 \mathrm{mg}$ taken three times daily. As AGI prevents the digestion of complex carbohydrates, they should be taken right at the start of the meal or with the first bite of the meal.

The most commonly encountered adverse effect is GI disturbances which is due to the nature of working of this particular class. However, flatulence is the most reported adverse effect of this class and is reported around $78 \%$ of the patients [20] [21]. 


\subsection{Meglitinides}

Drugs included are Nateglinide, Repaglinide, Mitiglinide.

The newer class of antidiabetic drug which works by increasing insulin secretion from the pancreas by blocking ATP sensitive potassium channels. This newer class of drug includes Repaglinide and Nateglinide by binding to a receptor different from sulfonylureas. These drugs have shorter duration of action and cleared in bile which makes them more suitable for Type 2 DM patients with more flexible lifestyles [20] [21]. The major adverse effects of Meglitinides are hypoglycemia which is due to increase in insulin secretion from the pancreas. The incidence of serious hypoglycemia is lower than that of sulfonylurea. It can also cause respiratory tract infections and headache just like sulfonylureas it also causes increase in weight. According to fda.gov Repaglinide causes an increase risk of benign adenomas especially of thyroid and liver in rats under studies, no such effect was seen with any of other drugs.

Meglitinides are contraindicated in patients with renal failure, in patients of diabetic ketoacidosis, type 1 diabetes mellitus, severe liver dysfunction and patients on gemfibrozil. It is also not recommended in patients over 75 years old in the USA

\subsection{DPP4 Inhibitors}

DPP4 inhibitors is a relatively newer group of anti diabetic medications used for the treatment of type II DM in patients, especially those who are overweight as it is associated with weight loss. Examples of drugs included in this class are Sitagliptin, Vitagliptin, Saxagliptin etc. [22].

DPP4 inhibitors as the name indicates inhibit the enzyme DPP4. DPP4 is responsible for the breakdown of incretins including Glucagon like Peptide 1 GLP1. Incretins are released by the intestinal cells after meals which increases insulin release from the pancreas in a glucose dependent manner. Incretins also inhibits glucagon release and slows gastric emptying [23]. DPP4 is used as alone therapy but sometimes can be combined with other agents such as Metformin and Sulfonylureas.

1) Type 1 Diabetes Mellitus

2) Diabetic ketoacidosis

DPP4 can be used alone or in patients who can not take or have contraindications to Metformin (e.g. CKD) or other oral anti diabetic medications. Also they can be used in combination with other classes of glucose lowering drugs (Sulfonylureas, SGLT2 inhibitors, Thiazolidinediaones) except GLP-1 agonists.

Studies done by the In Sweden in university of Lund, its clinical science department $0.6 \%-1.1 \%$ reduction of HbAlc occurred in studies followed up to 52 weeks. They are weight neutral and can be used in patients who are obese and in cases where weight loss is intended in addition to glycemic control. They can be used in early stages of Diabetes Mellitus type 2 in combination with Metformin to achieve adequate glycemic control [24]. 
According to a study done in Division of Diabetes, Nutrition and Metabolic Disorders, In Belgium at university of Liege, the reduction of HbA1c with DPP4 inhibitor was low as compared to Metformin and Sulfonylureas when used as monotherapy but it offered the advantage of better GI tolerability when compared with Metformin and No weight gain when compared with the later. Similarly study showed that combination of Metformin with Sitagliptin showed similar reduction of $\mathrm{HbA1c}$ if biguanides with Sulfonylureas if Biguanides with TZDs. Moreover, other clinical trials have shown that this class of drugs causes a significant reduction in $\mathrm{HbAlc}$ when combined with Insulin without increasing risk of hypoglycemia. However these drugs were less effective when compared with GLP1 agonists but they offered better compliance, being oral medication, and better affordability.

The most frequently encountered adverse effects include upper respiratory tract infections (URTIs), headaches, and naso pharyngitis [25] [26]. Since there are different potential di peptidyl peptidase substrates in the body, therefore the risk of side effects is considered to be higher in drugs with low specificity for DPP4.

There have been reported cases of acute pancreatitis with the use of DPP4 inhibitors and acute pancreatitis should be considered as a differential in patients having abdominal pain. For the same reason, a history of pancreatitis rules out the use of DPP4 inhibitors and should not be considered in such patients [27]. DPP4 inhibitors such as Vildagliptin and Alogliptin are associated with elevation of liver enzymes. Therefore it is necessary to evaluate LFTs prior to initiating therapy with these drugs and also at intervals of three months for the first year. Elevated Aspartate aminotransferase leads to discontinuation of these drugs [28]. According to a study based on the data collected from United Kingdom Clinical Practice Research Datalink, increased risk of Inflammatory Bowel Disease was noted with DPP4 inhibitors compared with other anti diabetic medications.

\subsection{Bromcriptine}

Dopamine release in hypothalamus inhibits release of many hormones such as growth hormones, prolactin, thyroid hormones and also activates dopamine receptors in basal ganglia causing movement. Similarly bromcriptine is dopamine receptor agonist and it inhibits release of excessive amount of growth hormones so treats Hyperglycemia of excessive growth hormones in patients of acromegaly, similarly in patients of secondary hyperthyroidism and hyper pro lactinemia there is also hyperglycemia when these high levels of prolactin (increase insulin resistance) and thyroid hormones occur in blood for years so to prevent this hyperglycemia, Bromcriptine is used. Bromcriptine is useful in Parkinson disease too because it acts on dopamine receptors in agonistic fashion and causes movements by completing basal ganglia neurotransmitters pathways by acting on naigro striatal pathways. In normal people the bromcriptine or dopamine in- 
creases release of growth hormones but in patients of growth hormone adenoma or acromegaly the bromcriptine decreases release of growth hormones paradoxically.

In the chemo trigger zone of the brain stem it activates the dopamine receptors causing nausea vomiting and constipation as an adverse effect so shall be used with caution in old age and pregnant ladies. It also causes hypotension by causing vasodilation because of inhibition of alpha adrenergic receptors when used in low doses, in high doses it can act like epinephrine as a vasoconstrictors and will cause hypertension. It also causes central nervous system excitation and is causing hallucinations, confusion and psychosis [29].

\subsection{Otelixizumab: (Also Known as TLX4)}

Diabetes mellitus type is an autoimmune disease characterized by infiltration of auto reactive $\mathrm{T}$ cells of beta cells of pancreas and causes inflammation, apoptosis and destruction of beta cells, leading to insulin deficiency in young age mostly. Apart from treatment of insulin and insulin analogues scientists are curious to treat this autoimmune disease by immune suppressive therapy and amongst them one is Otelixizumab. It inhibits auto reactive $\mathrm{T}$ cells by attaching to the epsilon chain of $\mathrm{CD} 3$ receptors, so in high doses it decreases CD3 receptor interactions with MHC proteins, CCR5 and CXCR4, so it limits the progression of type 1 diabetes mellitus. So simply it decreases reactivity of T cells [30]. It is also used in other autoimmune diseases such Rheumatoid arthritis and psoriasis.

It's an orphan drug according to food and drug regulation authority, side effects such as allergic reaction or autoimmune reaction occurs or increase risk to occur if used with other monoclonal antibody drug such Abciximab, Apiximab, Ritoximab, Anakinra, Bevacizumab etc. Studies on this drug are still under processing in clinical trial stage three.

\subsection{Recombinant Human Glutamic Acid Decarboxylase 65 (rhGAD65)}

Glutamic acid decarboxylase enzyme is present mainly in central nervous system, is a rate limiting enzyme for catalyzing the conversion of GABA from glutamate, a main inhibitory neurotransmitter for brain, but some quantity of this enzyme is present in the pancreatic beta cells. Antibodies against glutamic acid decarboxylase occur in a variety of diseases such as in diabetes mellitus type, and high quantities are associated with stiff person syndrome and cerebellar ataxia, also associated with autoimmune thyroiditis and pernicious anemia. Antibodies titer is measured by ELISA. Diabetes Mellitus type is an autoimmune disease characterized by autoantibodies against different varieties of antigens, four types of antibodies are found in type 1 diabetes mellitus.

\subsection{Autoantibodies against Glutamic Acid Decarboxylase}

Autoantibodies against insulin islet cells cytoplasmic autoantibodies Insulinoma 
associated 2 autoantibodies.

One of the major auto antigen against which auto antibodies form is glutamic acid decarboxylase enzyme 65. GAD-Alum is Recombinant human (rhGAD65) and is primarily used as an antigen-specific immune modulator. rhGAD65 is available in vaccine form to increase immune tolerance and as these antibodies are present in $80 \%$ to $90 \%$ of patients. When administered for two years there was reduction in $\mathrm{HbA} 1 \mathrm{C}$ and increase in secretion of insulin. There are three studies done which are two phased, done on people having age 10 years to 20 years, the objective of studies is to know whether the rhGAD65 which is formulated in alum fruit, mainatains the insulin secretions from beta cells normally or not, but the results will be available after few years.

Studies done in the past have shown that it may prevent or slow autoimmune destruction of the pancreatic islet cells by "immune tolerance". For example by administering excess autoantigen, the body may stop the mechanism by which it attacks on its own insulin producing. If the immune system's attack can be stopped in a recently diagnosed patient of T1DM, then residual insulin secretion can be maintained. These phenomena can be beneficial in decreasing acute and long-term complications of diabetes and also help in improving glucose control [31]. If this recombinant form is present in serum than antibodies will attach to it and will spare most of GAD of beta cells so the process of autoimmunity against beta cells will slow down, but studies on this are still in progress in clinical phase 2 and results won't be available for years so they are not available in market yet because they are not FDA approved.

\subsection{Succinobucol}

Succinobucol is taken per orally currently under process of development in clinical trial phase 3 for anti diabetic therapy, it is derivative of lipid lowering agent called probucol it is its mono succinate ester.

Atherosclerosis is complex process of lipid peroxidation of endothelium of blood vessels when lipid accumulate in macrophage and vascular smooth muscle cells, when diabetes hyperglycemic state and hypertension causes endothelial damage and lead to release of cytokines and vascular growth factors, vascular endothelial adhesion molecules that lead to progression of atherosclerosis. Succionobucol inhibits vascular adhesion molecules and its antioxidant inhibits lipids peroxidation so it prevents atherosclerosis in Diabetes mellitus type 2. It is in phase 3 clinical stage of development, so not approved by FDA for diabetes mellitus, and not available in market yet.

\subsection{Teplizumab}

It is humanized monoclonal antibody targets CD3 on T lymphocytes, reduces its signaling and so if these cytotoxic $\mathrm{T}$ lymphocytes are not stimulated for a long period and so it undergoes apoptosis, and regulatory $\mathrm{T}$ cells increases consecutively, so it's helpful in diabetes mellitus type which is autoimmune induced T 
cell mediated beta cells of pancreas damage. Once CD3 is inhibited on T effector cells, so no stimulatory signals for cytotoxic $\mathrm{T}$ cells and so no release of pro inflammatory cytokines such as TNF a, IL1, IL12. IL6 so effective in Diabetes mellitus type 1. Its efficacy is checked by glycemic control while using insulin, a lesser dose of insulin id required if Teplizumab is used for 6 months to one year in patients of age 5 years to 35 years of having diabetes mellitus type 1 .

Only side effects are we can not use it in diabetic patients who got infected by bacteria or virus, because these drugs suppress immunity so increase risk of sepsis and severe infections will occur. Already diabetic patients are immune compromised due to neutropenia from hyperglycemia so these immune compromising drugs are not always a safe options, just like cyclosporine once used for diabetes mellitus and then FDA disapprove it because of severe nephrotoxicity and imunocompromised state creation. These monoclonal antibodies are not easily available in the market and cost too high, available as IV form $10 \mathrm{ml}$ for 1200 US dollars.

\subsection{LY2189265}

It is a long acting GLP 1 analogue works just like incretin, lowers blood glucose by three mechanisms:

1) decrease gastric emptying so slow digestion less food intake

2) stimulates beta cells to release more insulin

3) inhibits alpha cells to decrease release of glucagon

It is used as adjunct therapy with Metformin to lower the glucose levels in type 2 diabetes mellitus. It causes a dose dependent increase in insulin secretion. The half life is approximately 90 hours and C max occurs between 24 to 48 hours in most subjects. There is evidence of increase in insulin secretion glucose dependence as compared to people on placebo during oral glucose tolerance tests and once a weekly dosing.

It is generally well tolerated with few Gastrointestinal side effects and cardiovascular side effects, in the GI system it causes Nausea, vomiting, and Pancreatitis with increase in satiety often desired. In cardiovascular side effects it causes an increase in pulse pressure slightly, if the dose is increased greater than $1 \mathrm{mg}$ and increase in diastolic pressure if dose is increased more than $3 \mathrm{mg}$ [32].

\subsection{AVE0010/ZP10 (Lixisenatide)}

A GLP 1 analogue used in diabetes mellitus type 2, once daily dosing, lowers the glycemic concentration in blood, by measuring HbA1C is lowered by $0.29 \%$ to $0.69 \%$ as compared to placebo. It is a long acting GLP 1 analogue works just like incretin, lowers blood glucose by three mechanisms:

1) decrease gastric emptying so slow digestion less food intake

2) stimulates beta cells to release more insulin

3) inhibits alpha cells to decrease release of glucagon.

It is generally well tolerated with few Gastrointestinal side effects and cardi- 
ovascular side effects, in the GI system it causes Nausea, vomiting, and Pancreatitis with increase in satiety often desired. In cardiovascular side effects it causes an increase in pulse pressure slightly, if the dose is increased greater than $1 \mathrm{mg}$ and increase in diastolic pressure if dose is increased more than $3 \mathrm{mg}$ [32].

All GLP1 analogues use reduced mortality by $12 \%$, hospital admission due to heart failure by $9 \%$, and progression to chronic kidney disease or progressive renal failure of end stage by $17 \%$ because it reduces micro albuminuria in diabetes mellitus, the main reason for this is reduction in albumin excretion while this mechanism also lead to increase in creatinine levels in serum. There is no risk of severe hypoglycemia or pancreatitis or pancreatic carcinoma [33].

\section{Conclusions}

Diabetes Mellitus is a globally progressive pandemic that affects people chronically from children to aged people, from mature people to pregnant ladies, to control the disease proper early diagnosis is immensely in need followed by proper treatment. Treatment strategies focus initially on diet, exercises, followed by use of drugs. Generally in older antidiabetic Sulfonylureas and Biguanides are well tolerated but some patients can not tolerate these drugs, like Biguanides are associated with nausea, vomiting, B12 deficiency and metallic taste while sulfonylureas causes severe hypoglycemia, weight gain and Disulfiram like reactions in alcoholic or patient on metronidazole, while Insulin are mostly daily injections apart from newer classes daily or twice a day, which is pretty embarrassing feeling for many patients, also causes hypoglycemia which is dangerous than hyperglycemia, weight gain and local fats blisters at injections sites. Older anti diabetics are easily available, cheap and widely used and highly studied drugs.

While newer Antidiabetics such as GLP 1 agonists increases insulin secretions has very low risk of hypoglycemia, causes weight loss as compared to insulin and decreases risk of cardiovascular side effects but still can't be used in renally impaired patients, causes pancreatitis and can not be given in gastroparesis patients, similarly a newer drug of this class known as LY2189265 has long halflife of 90 hours, better efficacy, but causes pancreatitis and increase diastolic BP in high doses, pancreatitis is not associated with lixisenatide (GLP 1 agonist), while DPP4 inhibitors which increases GLP 1 in body has less risk of hypoglycemia, GI side effects, are weight neutral can be used in CKD but causes headaches and Nasopharyngitis. Bromocriptine or pegvisomant are used in patients of growth hormones adenoma induced DM as a medical therapy but are associated with psychosis and hallucinations. Meglitinides increases insulin secretion and has minuscule risk of hypoglycemia but can not be used in CKD patients. Otelixizumab and Teplizumab decrease $\mathrm{T}$ cell functions and saves beta cells from immune reactions used in DM 1 but causes immune suppression and is an orphan drug. Recombinant GAD used in vaccines decreased antibody mediated beta cell damage but is still under studies. Succinobucol prevents atherosclerosis but is still under studies. Physicians shall focus more on recommendation of lifestyle 
modifications for control of such diseases and regular follow ups with hygiene. Social media programs, seminars on DM preventing strategies shall be increased by the governments of each country and globally working health NGOs.

\section{Funding}

SM is supported through the "Johns Hopkins-Afghanistan Pakistan International Collaborative Trauma and Injury Research Training program", [grant number D43-TW007292] from the Fogarty International Center of the United States National Institutes of Health. The content is solely the responsibility of the authors and do not represent the views of Fogarty or NIH.

\section{Conflicts of Interest}

The authors declare no conflicts of interest regarding the publication of this paper.

\section{References}

[1] Kumar, V., Abbas, A. and Aster, J. (2020) Robbins and Cotran Pathologic Basis of Diseases. 10th Edition, Elsevier, Amsterdam.

[2] Saeedi, P., Peterson, I., Salpea, P., Malanda, B., Karuranga, S., Unwin, N., et al. (2019) Global and Regional Diabetes Prevalence Estimates for 2019 and Projections for 2030 and 2045: Results from the International Diabetes Federation Diabetes Atlas, 9th Edition. Diabetes Research and Clinical Practice, 157, Article ID: 107843. https://doi.org/10.1016/j.diabres.2019.107843

[3] Centers for Disease Control and Prevention (2018) CDC Statistics of Diabetes in US 2018. https://www.cdc.gov/diabetes/pdfs/data/statistics/national-diabetes-sta

[4] Deborah, W. and Ncrna, R. (2019) Insulin Charts. Written by Heather Grey-Updated on March 4, 2019. https://www.healthline.com/health/type-2-diabetes/insulin-chart

[5] Villa, C., Pan, D., Zaitsev, S., Cines, D., Siegel, D. and Muzykantov, V. (2015) Delivery of Drugs Bound to Erythrocytes: New Avenues for an Old Intravascular Carrier. Therapeutic Delivery, 6, 795-826. https://doi.org/10.4155/tde.15.34

[6] Tao, L., Vikas, B. and Matthew, S. (2021) First Aid for the USMLE Step 12021.31 st Edition, McGraw-Hill Education, New York, 352-353.

[7] Mcfarthing, K., Larson, D. and Simuni, T. (2020) Clinical Trial Highlights-GLP-1 Agonists. Journal of Parkinson's Disease, 10, 355-368. https://doi.org/10.3233/JPD-200002

[8] Andreadis, P., Karagiannis, T., Malandris, K., Avgerinos, I., Liakos, A., Manolopoulos, A., et al. (2018) Semaglutide for Type 2 Diabetes Mellitus: A Systematic Review and Meta-Analysis. Diabetes, Obesity and Metabolism, 20, 2255-2263. https://doi.org/10.1111/dom.13361

[9] Berlie, H., Hurren, K.M. and Pinelli, N.R. (2012) Glucagon-Like Peptide-1 Receptor Agonists as Add-On Therapy to Basal Insulin in Patients with Type 2 Diabetes: A Systematic Review. Diabetes, Metabolic Syndrome and Obesity, 5, 165-174. https://doi.org/10.2147/DMSO.S27528

[10] Htike, Z.Z., Zaccardi, F., Papamargaritis, D., Webb, D.R., Khunti, K. and Davies, M.J. (2017) Efficacy and Safety of Glucagon-Like Peptide-1 Receptor Agonists in Type 2 Diabetes: A Systematic Review and Mixed-Treatment Comparison Analysis. Diabetes, Obesity and Metabolism, 19, 524-536. https://doi.org/10.1111/dom.12849 
[11] Marso, S.P., Daniels, G.H., Brown-Frandsen, K., Kristensen, P., Mann, J.F.E., Nauck, M.A., et al. (2016) Liraglutide and Cardiovascular Outcomes in Type 2 Diabetes. New England Journal of Medicine, 375, 311-322. https://doi.org/10.1056/NEJMoa1603827

[12] US Food and Drug Administration (2009) MedWatch: The FDA Safety Information and Adverse Event Reporting Program: Safety Information-Byetta (Exenatide)-Renal Failure.

http://www.fda.gov/Safety/MedWatch/SafetyInformation/SafetyAlertsforHumanMe dicalProducts/ucm 188703.htm

[13] Zinman, B., Wanner, C., Lachin, J.M., Fitchett, D., Bluhmki, E., Hantel, S., et al. (2015) Empagliflozin, Cardiovascular Outcomes, and Mortality in Type 2 Diabetes. New England Journal of Medicine, 373, 2117-2128. https://doi.org/10.1056/NEJMoa1504720

[14] Clar, C., Gill, J.A., Court, R. and Waugh, N. (2012) Systematic Review of SGLT2 Receptor Inhibitors in Dual or Triple Therapy in Type 2 Diabetes. BMJ Open, 2, Article No. e001007. https://doi.org/10.1136/bmjopen-2012-001007

[15] Liu, J., Li, L., Li, S., Wang, Y., Qin, X., Deng, K., et al. (2020) Sodium-Glucose Co-Transporter-2 Inhibitors and the Risk of Diabetic Ketoacidosis in Patients with Type 2 Diabetes: A Systematic Review and Meta-Analysis of Randomized Controlled Trials. Diabetes, Obesity and Metabolism, 22, 1619-1627.

https://doi.org/10.1111/dom.14075

[16] Derosa, G. and Maffioli, P. (2012) Mini-Special Issue Paper Management of Diabetic Patients with Hypoglycemic Agents $\alpha$-Glucosidase Inhibitors and Their Use in Clinical Practice. Archives of Medical Science, 8, 899-906.

https://doi.org/10.5114/aoms.2012.31621

[17] Bell, D.S., O’Keefe, J.H. and Jellinger, P. (2008) Postprandial Dysmetabolism: The Missing Link between Diabetes and Cardiovascular Events? Endocrine Practice, 14, 112-124. https://doi.org/10.4158/ep.14.1.112

[18] Yang, H.K., Lee, S.H., Shin, J., Choi, Y.-H., Ahn, Y.-B., Lee, B.-W., et al (2019) Acarbose Add-On Therapy in Patients with Type 2 Diabetes Mellitus with Metformin and Sitagliptin Failure: A Multicenter, Randomized, Double-Blind, Placebo-Controlled Study. Diabetes \& Metabolism Journal, 43, 287-301.

https://doi.org/10.4093/dmj.2018.0054

[19] Ceppa, E.P., Ceppa, D.P., Omotosho, P.A., Dickerson 2nd., J.A., Park, C.W. and Portenier, D.D. (2012) Algorithm to Diagnose Etiology of Hypoglycemia after Roux-en-Y Gastric Bypass for Morbid Obesity: Case Series and Review of the Literature. Surgery for Obesity and Related Diseases, 8, 641-647.

https://doi.org/10.1016/j.soard.2011.08.008

[20] Standl, E., Schernthaner, G., Rybka, J., Hanefeld, M., Raptis, S.A. and Naditch, L. (2001) Improved Glycaemic Control with Miglitol in Inadequately-Controlled Type 2 Diabetics. Diabetes Research and Clinical Practice, 51, 205-213. https://doi.org/10.1016/s0168-8227(00)00231-x

[21] Reuser, A.J. and Wisselaar, H.A. (1994) An Evaluation of the Potential Side-Effects of Alpha-Glucosidase Inhibitors Used for the Management of Diabetes Mellitus. European Journal of Clinical Investigation, 24, 19-24. https://doi.org/10.1111/j.1365-2362.1994.tb02251.x

[22] Ahrén, B. (2009) Clinical Results of Treating Type 2 Diabetic Patients with Sitagliptin, Vildagliptin or Saxagliptin-Diabetes Control and Potential Adverse Events. Best Practice \& Research Clinical Endocrinology \& Metabolism, 23, 487-498. https://doi.org/10.1016/j.beem.2009.03.003 
[23] Thornberry, N.A. and Gallwitz, B. (2009) Mechanism of Action of Inhibitors of Dipeptidyl-Peptidase-4 (DPP-4). Best Practice \& Research Clinical Endocrinology \& Metabolism, 23, 479-486. https://doi.org/10.1016/j.beem.2009.03.004

[24] Scheen, A.J. (2012) DPP-4 Inhibitors in the Management of Type 2 Diabetes: A Critical Review of Head-to-Head Trials. Diabetes \& Metabolism, 38, 89-101. https://doi.org/10.1016/j.diabet.2011.11.001

[25] Drucker, D.J. and Nauck, M.A. (2006) The Incretin System: Glucagon-Like Peptide-1 Receptor Agonists and Dipeptidyl Peptidase-4 Inhibitors in Type 2 Diabetes. Lancet, 368, 1696-1705. https://doi.org/10.1016/S0140-6736(06)69705-5

[26] Amori, R.E., Lau, J. and Pittas, A.G. (2007) Efficacy and Safety of Incretin Therapy in Type 2 Diabetes: Systematic Review and Meta-Analysis. JAMA, 298, 194-206. https://doi.org/10.1001/jama.298.2.194

[27] Singh, S., Chang, H.Y., Richards, T.M., Weiner, J.P., Clark, J.M. and Segal, J.B. (2013) Glucagon Like Peptide 1-Based Therapies and Risk of Hospitalization for Acute Pancreatitis in Type 2 Diabetes Mellitus: A Population-Based Matched Case-Control Study. JAMA Internal Medicine, 8, 534-539.

https://doi.org/10.1001/jamainternmed.2013.2720

[28] Gallwitz, B. (2019) Clinical Use of DPP-4 Inhibitors. Front Endocrinol (Lausanne), 10, 389. https://doi.org/10.3389/fendo.2019.00389

[29] Abrahami, D., Douros, A., Yin, H., Yu, O.H.Y., Renoux, C., Bitton, A., et al. (2018) Dipeptidyl Peptidase-4 Inhibitors and Incidence of Inflammatory Bowel Disease among Patients with Type 2 Diabetes: Population Based Cohort Study. BMJ, 21, Article No. k872. https://doi.org/10.1136/bmj.k872

[30] Guglielmi, C., Williams, S.R., Del Toro, R. and Pozzilli, P. (2016) Efficacy and Safety of Otelixizumab Use in New-Onset Type 1 Diabetes Mellitus. Expert Opinion on Biological Therapy, 16, 841-846. https://doi.org/10.1080/14712598.2016.1180363

[31] Tohid, H. (2016) Anti-Glutamic Acid Decarboxylase Antibody Positive Neurological Syndromes. Neurosciences (Riyadh), 21, 215-222. https://doi.org/10.17712/nsj.2016.3.20150596

[32] Barrington, P., Chien, J.Y., Tibaldi, F., Showalter, H.D.H., Schneck, K. and Ellis, B. (2011) LY2189265, a Long-Acting Glucagon-Like Peptide-1 Analogue, Showed a Dose-Dependent Effect on Insulin Secretion in Healthy Subjects. Diabetes, Obesity and Metabolism, 13, 434-438. https://doi.org/10.1111/j.1463-1326.2011.01365.x

[33] Kristensen, S.L., Rørth, R., Jhund, P.S., Docherty, K.F. Sattar, N., Preiss, D., et al. (2019) Cardiovascular, Mortality, and Kidney Outcomes with GLP-1 Receptor Agonists in Patients with Type 2 Diabetes: A Systematic Review and Meta-Analysis of Cardiovascular Outcome Trials. Lancet Diabetes \& Endocrinology, 7, 776-785. https://doi.org/10.1016/S2213-8587(19)30249-9

\section{Abbreviations}

DM: diabetes mellitus

DPP4: dipeptidyl peptidase 4

SGLT: sodoium glucose transporters on nephron

GLP: glucagon like peptide 